\title{
Spectral Efficiency and Fairness Tradeoffs in Cellular Networks with Realtime+Nonrealtime Traffic Mix using Stochastic Petri Nets
}

\author{
Rainer Schoenen*, Akram Bin Sediq*, Halim Yanikomeroglu*, Gamini Senarath ${ }^{\S}$, Zhijun Chao ${ }^{\S}$, and Ho Ting Cheng§ \\ *Department of Systems and Computer Engineering, Carleton University, Ottawa, Ontario, Canada \\ $\S$ Huawei Technologies Canada Co., LTD.
}

\begin{abstract}
Resource scheduling in OFDMA cellular wireless networks is a powerful technique on the MAC layer. Utilizing adaptive modulation and coding allows the effective use of all signal-to-interference ratio (SINR) ranges. Typical single antenna spectral efficiency values for LTE-Advanced range between 4.8 near the base station and $0.2 \mathrm{~b} / \mathrm{s} / \mathrm{Hz}$ at the cell edge. With besteffort traffic and full buffer assumption the tradeoff between emphasizing the cell center or cell edge can be explored extensively. However, with real-time traffic present, this takes priority without fairness adjustment alternatives. In this paper the mixed traffic scenario is studied with an abstract stochastic Petri net model. The exploration of the degrees of freedom by studying the real-time traffic proportion and a fairness adjustment parameter provides new insight to the potential feasible region. The results show that the tradeoff between emphasizing the cell edge performance and maintaining a high average spectral efficiency is most powerful in the best-effort case, while an increasing level of real-time traffic reduces the room for a tradeoff. The stochastic Petri net analysis approach allows numeric analysis without simulation by utilizing Markov chain equivalence and steady state calculations. This model is deliberately abstract but flexible enough to study the tradeoff.
\end{abstract}

Index Terms-cellular; traffic mix; real-time traffic; fairness; stochastic Petri nets; spectral efficiency

\section{INTRODUCTION}

$\mathbf{T}$ HE traffic mix in wireless networks makes a difference between pure physical layer (PHY) and medium access control (MAC) layer analysis. In PHY, features like adaptive modulation and coding (AMC) on all subchannels of OFDMA can adapt to variations of the SINR. This translates into a variation of local spectral efficiency $\gamma$ in the range of 0.2 to $4.8 \mathrm{~b} / \mathrm{s} / \mathrm{Hz}$ (LTE-A) from the cell edge to the center. Figure 1(a) shows the typical AMC modes of LTE-Advanced, their performance and the upper bounded given by the Shannon capacity. Figure 1(b) shows the probability of each AMC mode in the Urban Macro (UMa) cellular scenario (as defined by IMT-Advanced evaluation guidelines [1]). Numbers have been obtained using numeric analysis [2] for sufficiently many location points in the coverage area.

Previous work using the same approach has studied resource scheduling in a cell for non-realtime traffic (NRT) only [3]. In this study the different resource scheduling goals were analyzed, expecially the tradeoff between high spectral efficiency averaged over the whole cell area $(\bar{\gamma})$ and the cell edge performance $\gamma_{C E}$, using a parameter to adjust between MaxSINR (MS) and generalized proportional fair (GPF) schedul- ing. This tradeoff is only valid for non-realtime traffic (NRT), e.g., best-effort (BE), because of its elasticity and reasonably acceptable assumption of full buffers. With additional realtime traffic (RT), those RT packets have to be delivered while maintaining the ingress rate, or extensive packet loss (overly delayed packets) would occur. In this paper, a traffic mix of RT ant BE traffic is studied, and the potential tradeoffs and fairness implications are explored.

This paper contributes an abstract simulation-free and toolsupported model based on stochastic Petri nets (SPN), in contrast to traditional evaluation methods that require simulation and detailed scheduler implementations. Due to the equivalence of Markov chains and SPN state space, a numeric analysis can calculate steady state probabilities and easily obtain reward measures. The traffic mix (RT to NRT) is determined by a parameter $r$ and a tuning parameters in this model allows to explore the tradeoff between $\bar{\gamma}, \gamma_{C E}$ and fairness $J$ (either for rate or resources).

The advantage of Petri nets [4] is the abstract yet powerful way for modeling using a graphical structure that unfolds into a Markov chain (MC) of arbitrary connectivity and complexity with a few graph elements. Generalized SPN (GSPN) [5] is a useful tool, because results are obtained by matrix numeric tools without any simulation. GSPN analysis is well supported by tools [6]. GSPN have been used in the recent years to model communications systems [7], [8] and protocols [9]. Resource management in cellular wireless systems [10], multihop (relay) transmissions [11] and IEEE 802.16 [12] have also been studied using the SPN approach. The wireless channel can be modeled with SPN [11]. Even TCP models [13] and CreditBased Flow Control [14] can be integrated. Nobody has come up with an abstract SPN model like in this paper, hence the novelty is the SPN modeling itself.

The paper is organized as follows: Section II describes the radio cell model with multi-class traffic and the fairness versus rate objective. The last section III shows performance results for spectral efficiency and fairness.

\section{AN SPN MODEL FOR RESOURCE SCHEDULING OF RT+NRT TRAFFIC}

For an introduction into stochastic Petri nets, [4] and [5] are highly recommended. Due to space limitations we cannot introduce SPN here. Figure 2 shows the SPN model for a 


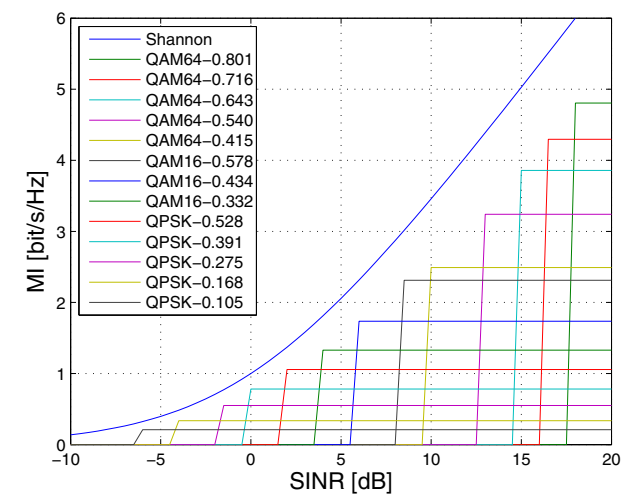

(a) Adaptive Modulation and Coding (AMC) performance.

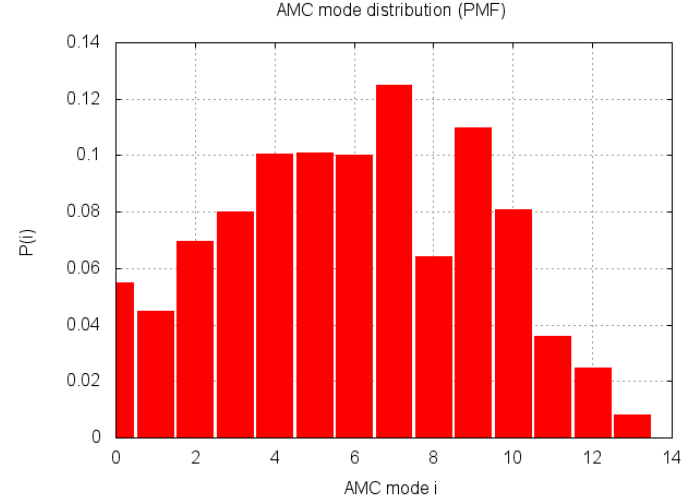

(b) LTE-A AMC probability $\pi_{i}$ in unconstrained UMa scenario.

Fig. 1. The underlying system model is completely described by the probability $\pi_{i}$ of each AMC mode. The values have been obtained by a previous LTE-Advanced simulations with 19 cells, UMa scenario, wraparound, including shadowing, fading, dual path loss, and 100 drops of random placement of 10 UTs per sector [1]. These baseline results were obtained using a proportional fair resource scheduler without traffic classes.

traffic mix of RT and NRT traffic. It extends a previous work in [15], which treated NRT traffic only. The places P\#\# model the user terminal (UT) selection of the scheduler: A token in one of these places represents a UT where this AMC mode index \#\# has been selected. P00 represents a temporary outage, i.e., no available AMC mode. According to Figure 1(a) this is a translation from the SINR distribution. In Figure 2 the AMC(UT) selection is modeled by probabilistic weights $w_{i}$ on the transitions $A M C 00$ to $A M C 13$. In an unconstrained operation, the weights are simply defined as $w_{i}=\pi_{i}$, the known occurrence from Figure 1(b) and representative for a resource fair situation. These probabilities $\pi_{i}$ were obtained before by simulation [1] in the UMa IMT-A scenario with realistic conditions, including interference, shadowing [3] and a proportional fair scheduler. The timing behavior is determined by transition Timer, and the rest of the SPN is constructed to conserve (limit) the token count in a loop, so that the PN is bounded, live and the Markov chain has a limited number of states. This model abstracts from (a limited integer count of) individual users but instead the model corresponds to a continuous user density ( $\infty$ users) in the cell area. The model also represents the full buffer situation (all UTs have packets available to send) and all OFDMA resource blocks (RBs) are in use. No individual RBs are modeled (time averaged).

The weights for the NRT scheduling selection with $A M C \# \#$ in Figure 2 are adjustable by a parameter, so that the different goals can be traded off. In a Proportional Fair scheduler the temporary dynamic priority of a connection $i$ is given by

$$
P_{i}=\frac{\gamma_{i}^{h}}{H_{i}}
$$

where $\gamma_{i}$ is the currently assumed $\operatorname{AMC}(i)$ and $H_{i}$ is the historical average rate of UT $i$. The weights $w_{i}$ in the SPN are now defined as:

$$
w_{i}^{B E}=\pi_{i} \cdot \gamma_{i}^{h} .
$$

It has been shown that the exponential emphasis by parameter $h \in[-2 ; 2]$ can scale between different fairness objectives [15]. With $h$ the UT selection can be biased proportional to $\gamma_{A M C}(h=1)$, independent of $\gamma_{A M C}(h=0)$, or inversely

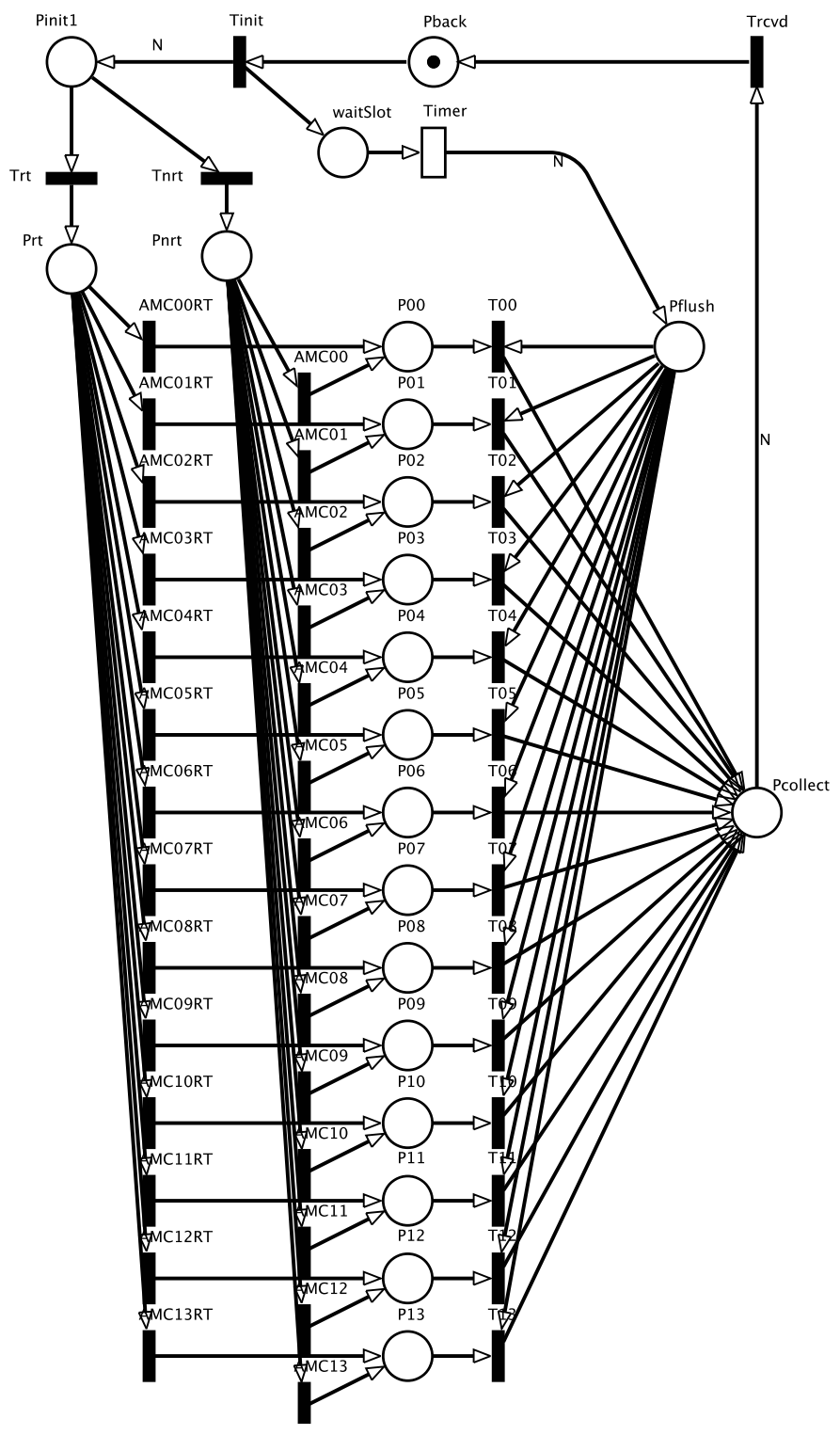

Fig. 2. Stochastic Petri Nets of the analyzed UMa scenario in the multitraffic scenario (RT and NRT). The places P\#\# model the UT selection of the scheduler, more precisely an arbitrary UT out of the cell area fraction where the average AMC mode index is \#\#. The AMC\#\# transitions schedule NRT and AMC\#\#RT schedule RT traffic. 


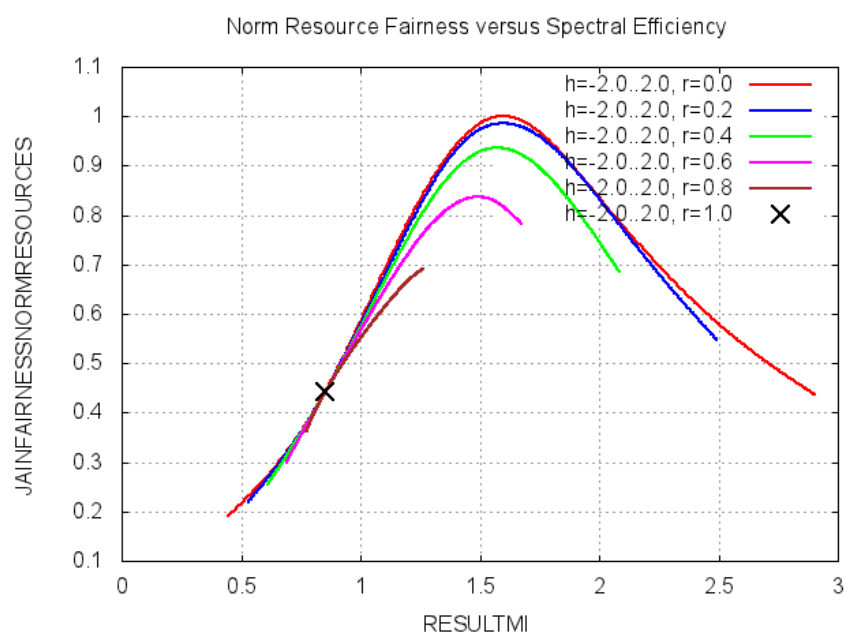

Fig. 3. Resource fairness $J_{S}$ versus $\bar{\gamma}$ in $\mathrm{b} / \mathrm{s} / \mathrm{Hz}$.

proportional to $\gamma_{A M C}(h=-1)$. More extreme emphasis is possible by any real $|h|>1$ or $\propto \sqrt{\gamma}$ by $h=\frac{1}{2}$. $h=1$ gives preference to UTs with high $\gamma$, so $\bar{\gamma}$ is expected to be high at the expense of $\gamma_{C E}$ and fairness performance. $h=-1$ gives more resources to cell edge UTs, therefore $\bar{\gamma}$ decreases while $\gamma_{C E}$ is increased. This fairness tradeoff is relevant only for elastic BE traffic, because RT traffic has to be delivered to the UT without noticeable loss or delay. This will be visible in the results.

Figure 2 models the scenario with a variable proportion $r$ of RT traffic. The proportion is installed by weights $r$ and $(1-r)$ at the transitions $T_{r t}$ and $T_{n r t}$. RT transmission requests (modeled by a token in $P_{r t}$ ) are dispatched through the transitions $A M C 00 R T$ to $A M C 13 R T$. Their weighted scheduling differs from BE traffic in Eq. 2 by the fact, that the rate must be guaranteed. Therefore a resource with weaker AMC mode $\gamma_{i}$ must be assigned an inversely proportional number of resources to guarantee the requested rate:

$$
w_{i}^{R T}=\pi_{i} \cdot \gamma_{i}^{-1}
$$

Both BE and RT contribute to the consumption of resources in the different AMC levels. With more RT traffic and its priority over BE traffic, the influence of the fairness control with $h$ diminishes. The analysis results in the next section show the tradeoff and results for $\bar{\gamma}, \gamma_{C E}$ and a fairness assessment.

Fairness is commonly defined by the Jain's fairness index:

$$
J(\vec{r})=\frac{\left(\sum_{i=1}^{N} r_{i}\right)^{2}}{N \cdot \sum_{i=1}^{N} r_{i}^{2}} .
$$

It can be used to evaluate scheduling fairness among different UTs. $J_{R}$ is defined as rate fairness when it compares data rates $r_{i}$ per AMC region index $i$, and as resource fairness $J_{S}$ when comparing the number of assigned RB resources $R_{i}$.

\section{PERformance AnAlysis}

Using tool support [6] for generating the Markov chains and the stationary state probabilities, parameterized experiments

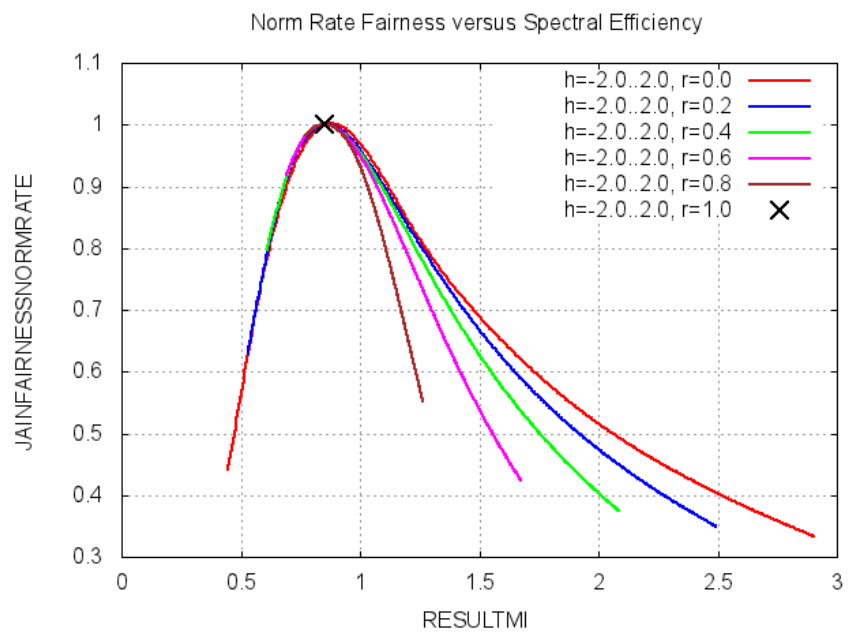

Fig. 4. Rate fairness $J_{R}$ versus $\bar{\gamma}$ in $\mathrm{b} / \mathrm{s} / \mathrm{Hz}$.

can be run and analyzed. The reward measures are obtained from the token distribution $p_{i}$ :

$$
\bar{\gamma}=\sum_{i=0}^{13} p_{i} \cdot \gamma_{i}, \quad \gamma_{C E}=\operatorname{Pr}(\# P 01) \cdot \gamma_{01} .
$$

A fairness metric is obtained separately for rate (Eq. 6) and resources (Eq. 7). It is normalized taking the probability $\pi_{i}$ in each AMC mode $i$ into account. With homogeneous UT density $p_{i} \cdot \pi_{i}^{-1}$ equals one for a fair assignment.

$$
\begin{gathered}
J_{R}=\frac{\left(\sum_{i=1}^{13} p_{i} \cdot \pi_{i}^{-1} \cdot \gamma_{i}\right)^{2}}{13 \cdot \sum_{i=1}^{13}\left(p_{i} \cdot \pi_{i}^{-1} \cdot \gamma_{i}\right)^{2}}, \\
J_{S}=\frac{\left(\sum_{i=1}^{13}\left(p_{i} \cdot \pi_{i}^{-1}\right)\right)^{2}}{13 \cdot \sum_{i=1}^{13}\left(p_{i} \cdot \pi_{i}^{-1}\right)^{2}} .
\end{gathered}
$$

The analysis of the SPN in Figure 2 provides detailed numeric results (at the end of this paper in Figure 7). Without scheduler tuning and BE traffic only, the reference values are $\bar{\gamma}=1.58 \mathrm{~b} / \mathrm{s} / \mathrm{Hz}, \gamma_{C E}=0.0094 \mathrm{~b} / \mathrm{s} / \mathrm{Hz}, J_{R}=0.680$ and $J_{S}=1.0$ (a resource=proportional fair assignment). The parameter analysis now varies the RT traffic proportion $r$ and for the remaining $(1-r) \cdot 100 \% \mathrm{BE}$ traffic, $h$ is adjusted to explore the tradeoff. As result of this analysis, the impact on the two fairness metrics $J_{R}$ and $J_{S}$ and spectral efficiencies $\bar{\gamma}$ and $\gamma_{C E}$ are obtained as a function of the two parameters $r$ and $h$.

With BE traffic only $(r=0)$ the results in Fig. 7(a) show that $h$ can be used to adjust the cell spectral efficiency $\bar{\gamma}$ at the expense of the cell edge performance $\gamma_{C E}$ in Fig. 7(c). The same insight can be seen in Fig. 7(b) and 7(d) on the left margin $(r=0)$. The other extreme is with $r=1$ (only $\mathrm{RT}$ traffic). In this case there is no tradeoff possible and both $\bar{\gamma}$ and $\gamma_{C E}$ stay constant (can be seen in Fig. 7(a) to Fig. 7(d)). Interestingly, when $r$ is gradually increased, as shown in Fig. 7(b) and 7(d), the reduction in $\bar{\gamma}$ and increase in $\gamma_{C E}$ happen linearly in $r$. In the same direction of increasing $r$ the rate fairness $J_{R}$ approaches its maximum and the resource fairness $J_{S}$ reduces to 0.45 . Note that $J_{S}$ has been perfect 1.0 for the reference case (proportional fair for $\mathrm{BE}, h=0$ ). 


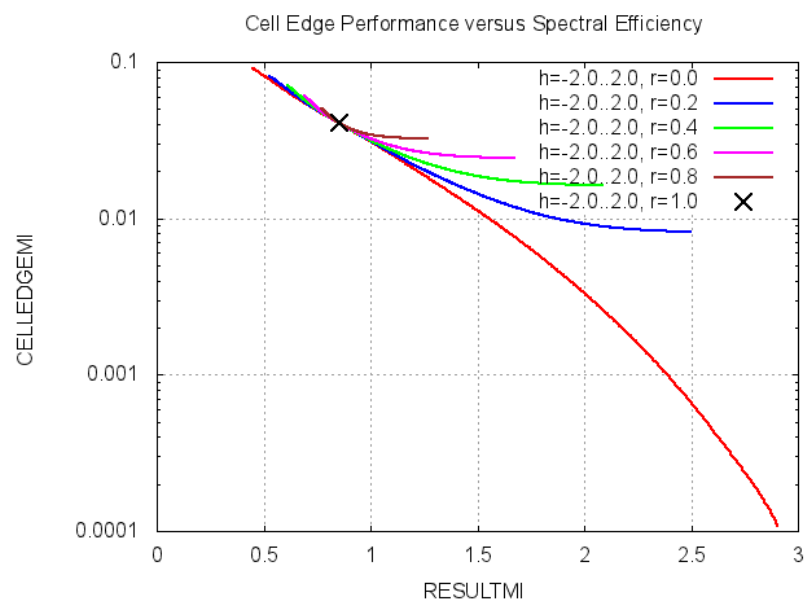

Fig. 5. Tradeoff between average spectral efficiency $\bar{\gamma}$ (x-axis, in b/s/Hz) and cell edge performance $\gamma_{C E}(\mathrm{~b} / \mathrm{s} / \mathrm{Hz})$ for an increasing proportion of RT traffic $(r)$. At $r=1$ there is no more flexibility, therefore one point (X) only.

Obviously the degree of freedom with $h$ shrinks with more and more RT traffic. Rate fairness $J_{R}$ in Fig. 7(e) is optimum for $h=-1$, which means basically the same scheduling treatment for $\mathrm{BE}$ as for RT traffic. Resource fairness $J_{S}$ in Fig. 7(f) is optimum for $h=0$ and $r=0$, i.e., only achievable with $\mathrm{BE}$ traffic due to its elasticity. A small proportion of RT traffic $(r=0.2)$ does not change much, but full RT load is bad for resource fairness, however good for rate fairness (Fig. 7(e), 7(f)).

The fairness objective can be analyzed as a function of the cell spectral efficiency (with parameters $r$ and $h$ ). This is displayed in Fig. 3 for resource fairness and Fig. 4 for rate fairness. Clearly the optimum resource fairness $J_{S}$ is achievable only for $r=0$, whereas $r=1$ is a fixed point $(\bar{\gamma}=0.8 \mathrm{~b} / \mathrm{s} / \mathrm{Hz})$. Rate fairness $J_{R}$ in Fig. 4 is optimum for $r=1$, but for $r<1$ this only works for a specific parameter of $h$, precisely $h=-1$.

The main result is Fig. 5, because it shows the tradeoff between $\bar{\gamma}$ and $\gamma_{C E}$ in one graph. For $r=0$, the balance between cell spectral efficiency and cell edge performance can fully be explored by $h$. This can actually be compared to other results in the literature [3]. However, the more RT traffic is accepted, the less flexibility is available. For $r \rightarrow 1$, the parametric plot converges to a fixed point at $\bar{\gamma}=0.8$ and $\gamma_{C E}=0.04$ (marked with $\times$ ).

Figure 6 shows the possible achievable rates (normalized to $1 \mathrm{~Hz}$ bandwidth) for NRT and RT, if mixed between $0 \%$ and $100 \%$ (implicit parameter $r$ ). The area below the lines is the schedulable region. The maximum NRT throughput depends on the parameter $h$ which trades off cell edge and average spectral efficiency. With $h=2$, NRT performance comes with an extremely unfair cell center preference over the cell edge.

The results also show that the abstract SPN model is powerful enough to provide performance estimates for real systems, which otherwise have to be modeled and analyzed by extensive simulation studies. Note that this level of abstraction allows understanding the root cause for the tradeoffs discussed here but it cannot model details of finite \#RBs, finite \#UTs and fading in time and frequency.

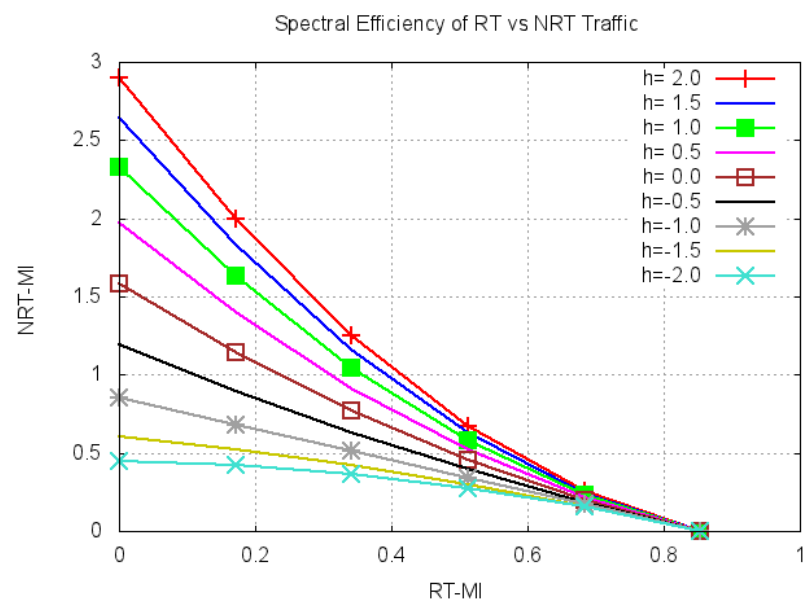

Fig. 6. Schedulable region with NRT and RT traffic. The spectral efficiency values along the axes denote the potential capacity $(\mathrm{b} / \mathrm{s} / \mathrm{Hz})$ of the corresponding traffic class, if mixed between $0 \%$ and $100 \%$ ratio. The parameter $h$ adjusts the fairness target for NRT traffic.

\section{CONCLUSION}

This paper treats the potential degree of freedom by a scheduling tradeoff between average cell spectral efficiency, cell edge performance and fairness in cellular networks with a traffic mix of realtime (RT) and non-realtime (NRT) traffic. The system is abstractly modeled as a stochastic Petri net (SPN) which incorporates a parameter that models the tuning parameter of a real scheduler. Results were obtained by Markov chain steady state analysis, not by simulation. The numbers show that a tradeoff is possible only for NRT traffic, but with increasing proportion of RT traffic, this flexibility shrinks down to zero. Also, spectral efficiency results must be assumed much lower, the more RT traffic is present. The transport of RT traffic must guarantee the equality of ingress and egress rate, therefore the cell spectral efficiency is lower for RT than for NRT traffic with (resource) proportional fairness. The contributed SPN model allows abstract and fast performance studies without the need of simulations. Thus this novel approach is the main contribution of this paper. Future work can include more classes and a variety of more schedulers. Adding active fading by channel variation models [11] in another potential extension.

\section{REFERENCES}

[1] ITU, "Report ITU-R M2135-1; Guidelines for evaluation of radio interference technologies for IMT-Adcanced", 2009.

[2] M. Mühleisen, D. Bültmann, and R. Schoenen, "Analytical Validation of an IMT-Advanced Compliant LTE System Level Simulator," in Proceedings of the European Wireless, Vienna, Apr 2011.

[3] A. B. Sediq, R. Schoenen, H. Yanikomeroglu, G. Senarath, and Z. Chao, "A novel distributed inter-cell interference coordination scheme based on projected subgradient and network flow optimizations," in PIMRC'2011, Toronto, Canada, Sep 2011.

[4] T. Murata, "Petri nets: Properties, analysis and applications," Proceedings of the IEEE, vol. 77, no. 4, pp. 541-581, April 1989.

[5] M. Marsan, Modelling with generalized stochastic Petri nets. Wiley, 1996, ISBN 0-471-93059-8.

[6] R. German, "A toolkit for evaluating non-markovian stochastic Petri nets," Performance Evaluation, vol. 24, pp. 69-87, 1995.

[7] J. Billington et al., Application of Petri Nets to Communication Networks. Springer, 1999, ISBN 3-540-65870-X. 


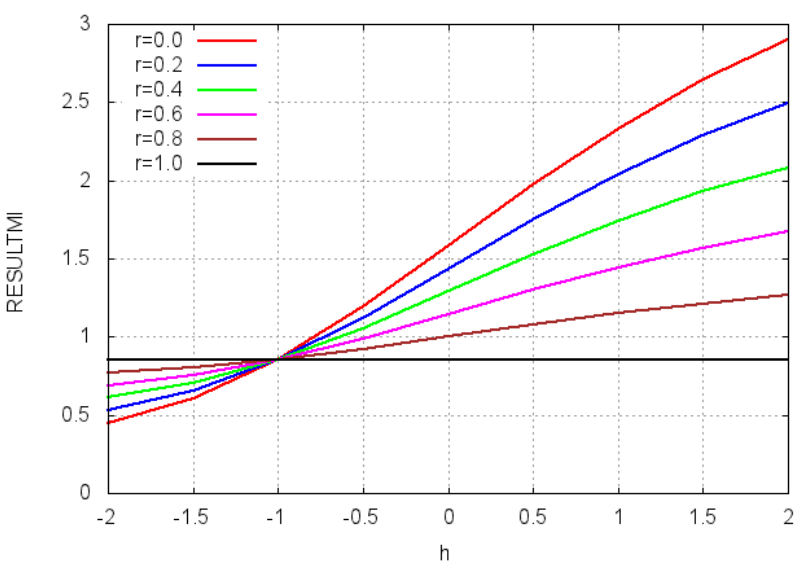

(a) Average spectral efficiency $\bar{\gamma}=f(\underline{\mathbf{h}}, r)$ in $\mathrm{b} / \mathrm{s} / \mathrm{Hz}$.

CELLEDGEMl=f(h)

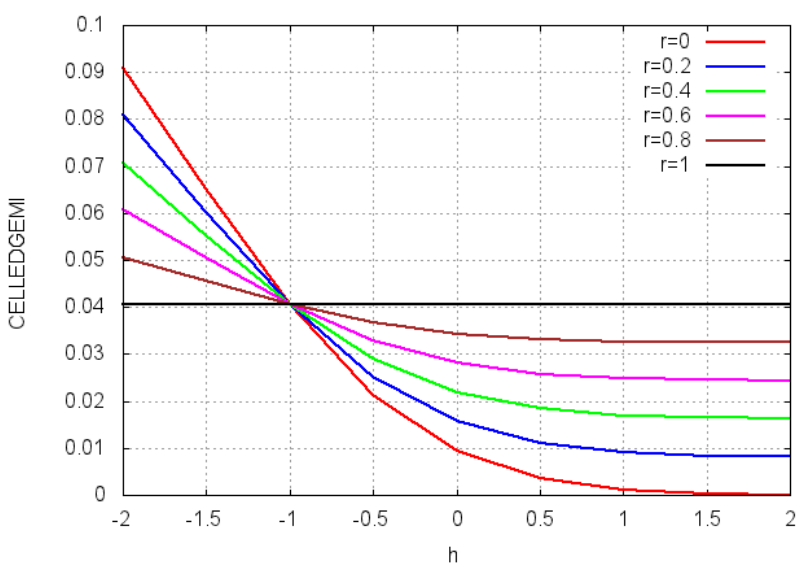

(c) Cell edge performance $\gamma_{C E}=f(\underline{\mathbf{h}}, r)$ in $\mathrm{b} / \mathrm{s} / \mathrm{Hz}$.

JAINFAIRNESSNORMRATE $=f(h)$

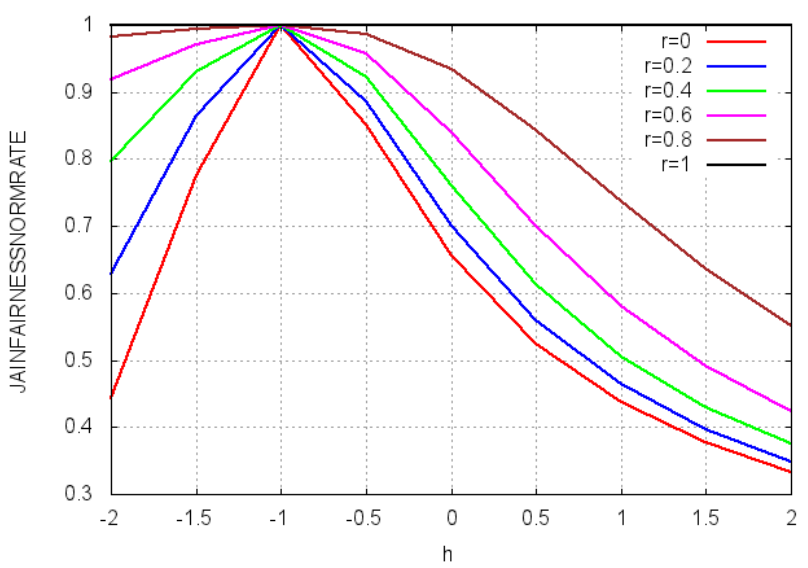

(e) Rate fairness $J_{R}=f(\underline{\mathbf{h}}, r)$.

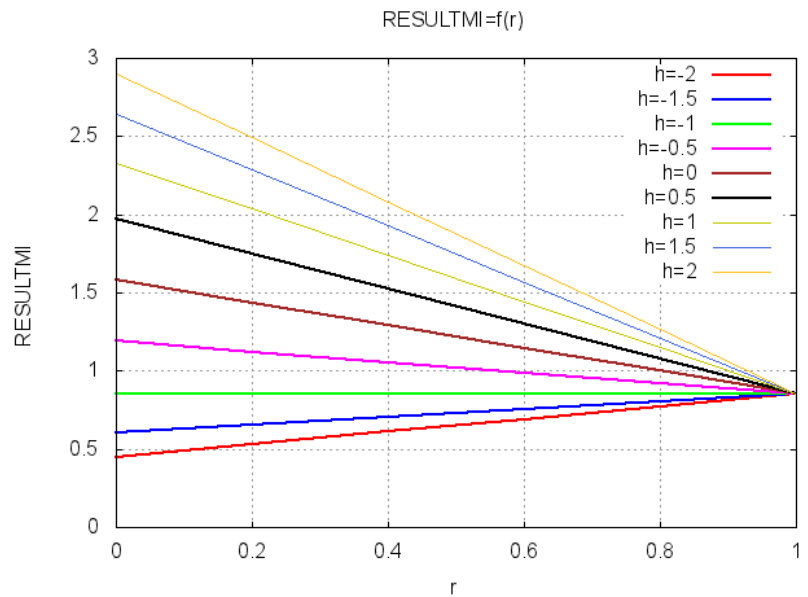

(b) Average spectral efficiency $\bar{\gamma}=f(h, \underline{\mathbf{r}})$ in $\mathrm{b} / \mathrm{s} / \mathrm{Hz}$.

CELLEDGEMl $=f(r)$

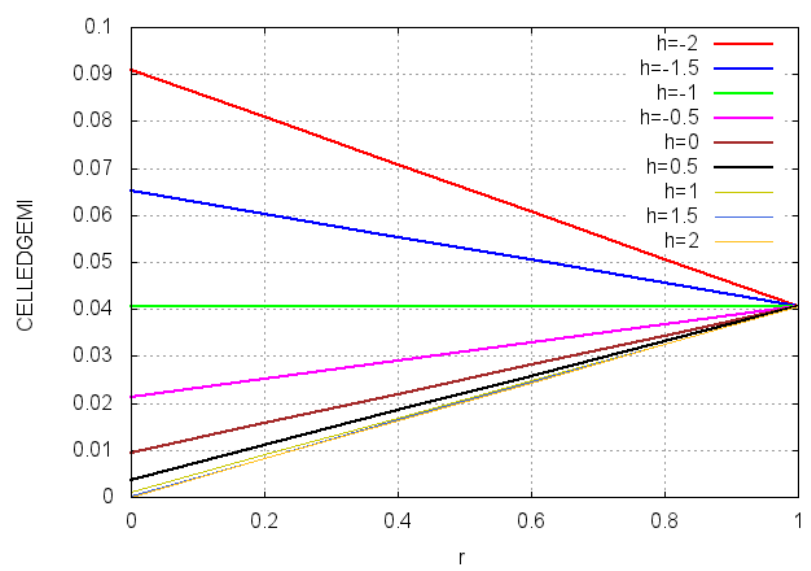

(d) Cell edge performance $\gamma_{C E}=f(h, \underline{\mathbf{r}})$ in $\mathrm{b} / \mathrm{s} / \mathrm{Hz}$.

JAINFAIRNESSNORMRESSOURCES $=\mathrm{f}(\mathrm{h})$

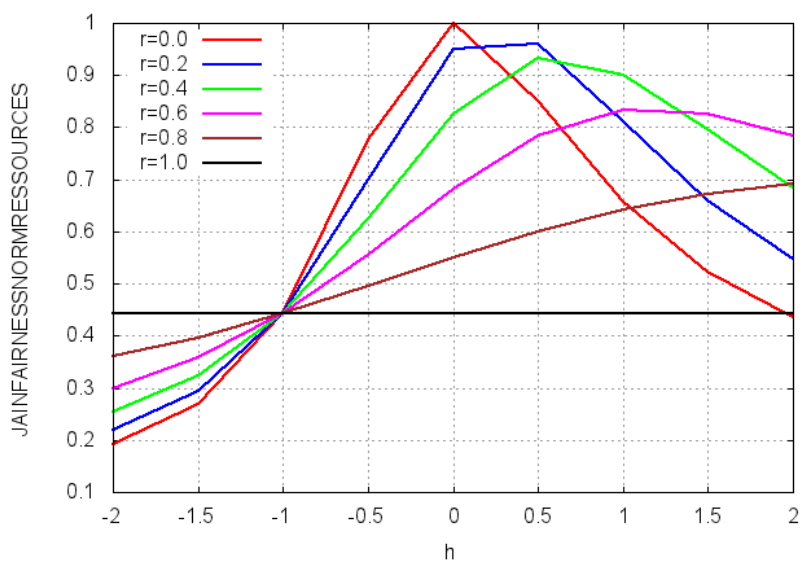

(f) Resource fairness $J_{S}=f(\underline{\mathbf{h}}, r)$.

Fig. 7. Average and cell edge spectral efficiency results $\left(\bar{\gamma}\right.$ and $\left.\gamma_{C E}\right)$ and rate fairness results $J_{R}$ depending on scheduling parameters $h$ and the RT traffic proportion $r$. The results converge for $r \rightarrow 1$, so that no tradeoff can be exploited when there is only RT traffic. As a consequence, spectral efficiency values for any wireless system which were obtained for elastic NRT traffic and proportional-fair scheduling become much lower with increasing RT traffic proportion.

[8] L. Lei, C. Lin, J. Cai, and X. Shen, "Performancs analysis of wireless opportunistic schedulers using stochastic Petri nets," IEEE Transactions on Wireless Communications, vol. 8, no. 4, April 2009.

[9] M. Bosch and G. Schmid, "Generic petri net models of protocol mechanisms in communication systems," Computer Communications, vol. 14 , no. 3 , pp. 143 - 156, 1991 .

[10] F. Jaimes-Romero, D. Munoz-Rodriguez, C. Molina, and H. Tawfik, "Modeling resource management in cellular systems using Petri nets," in IEEE Transactions on Vehicular technology, May 1997, pp. 298-312.

[11] R. Schoenen, M. Salem, A. Sediq, and H. Yanikomeroglu, "Multihop wireless channel models suitable for stochastic Petri nets and markov state analysis," in Proc. IEEE VTC Spring, Budapest, May 2011.

[12] S. Geetha and R. Jayaparvathy, "Modeling and analysis of bandwidth allocation in IEEE 802.16 MAC: A stochastic reward net approach,' Int. J. Communications, Network ans System Sciences, vol. 3, no. 7, pp. 631-637, July 2010.

[13] R. Gaeta, M. Gribaudo, D. Manini, and M. Sereno, "On the use of Petri nets for the computation of completion time distribution for short TCP transfers," Proceedings of the 24th international conference on Applications and theory of Petri nets, vol. LNCS, pp. 181-200, 2003.

[14] R. Schoenen, "Credit-based flow control for multihop wireless networks and stochastic Petri nets analysis," in Proceedings of the CNSR, Ottawa, May 2011.

[15] R. Schoenen, A. B. Sediq, H. Yanikomeroglu, G. Senarath, and Z. Chao, "Fairness analysis in cellular networks using stochastic Petri nets," in Proceedings of PIMRC'2011, Toronto, Canada, Sep 2011. 